This is an electronic reprint of the original article. This reprint may differ from the original in pagination and typographic detail.

Author(s): Gigli, Nicola; Mondino, Andrea; Rajala, Tapio

Title: $\quad$ Euclidean spaces as weak tangents of infinitesimally Hilbertian metric measure spaces with Ricci curvature bounded below

Year: $\quad 2015$

Version:

Please cite the original version:

Gigli, N., Mondino, A., \& Rajala, T. (2015). Euclidean spaces as weak tangents of infinitesimally Hilbertian metric measure spaces with Ricci curvature bounded below. Journal für die reine und angewandte Mathematik, 2015(705), 233-244. https://doi.org/10.1515/crelle-2013-0052

All material supplied via JYX is protected by copyright and other intellectual property rights, and duplication or sale of all or part of any of the repository collections is not permitted, except that material may be duplicated by you for your research use or educational purposes in electronic or print form. You must obtain permission for any other use. Electronic or print copies may not be offered, whether for sale or otherwise to anyone who is not an authorised user. 


\title{
Euclidean spaces as weak tangents of infinitesimally Hilbertian metric measure spaces with Ricci curvature bounded below
}

\author{
By Nicola Gigli at Nice, Andrea Mondino at Zurich and Tapio Rajala at Jyväskylä
}

\begin{abstract}
We show that in any infinitesimally Hilbertian $\mathrm{CD}^{*}(K, N)$-space at almost every point there exists a Euclidean weak tangent, i.e., there exists a sequence of dilations of the space that converges to a Euclidean space in the pointed measured Gromov-Hausdorff topology. The proof follows by considering iterated tangents and the splitting theorem for infinitesimally Hilbertian $C D^{*}(0, N)$-spaces.
\end{abstract}

\section{Introduction}

Gromov-Hausdorff limits of Riemannian manifolds with Ricci curvature lower bounds, Ricci-limits for short, have been extensively studied in particular by Cheeger and Colding in a series of papers [9-15]. In [10] they proved, among other things, that the tangent space at almost every point - intended as pointed Gromov-Hausdorff limit of rescaled spaces - of a Ricci-limit space is Euclidean, with dimension possibly depending on the point. Only much later, in [16] Colding and Naber showed that in fact for any Ricci-limit space there exists $k \in \mathbb{N}$ such that at almost every point in the space the tangent cone is $\mathbb{R}^{k}$. Notice however, that there can be points in the Ricci-limits where the tangent is not unique, see for instance [17] for examples.

Lott and Villani [27] and Sturm [32,33] independently proposed a definition of 'having Ricci curvature bounded from below by $K$ and dimension bounded above by $N$ ' for metric measure spaces, these being called $\operatorname{CD}(K, N)$-spaces (in [27] only the cases $K=0$ or $N=\infty$ were considered). Here $K$ is a real number and $N$ a real number at least one, the value $N=\infty$ being also allowed.

The crucial properties of their definition are the compatibility with the smooth Riemannian case and the stability with respect to measured Gromov-Hausdorff convergence.

More recently, Bacher and Sturm [5] proposed a variant of the curvature-dimension condition $\mathrm{CD}(K, N)$, called reduced curvature-dimension condition and denoted as $\mathrm{CD}^{*}(K, N)$ which, while retaining the aforementioned stability and compatibility, has better globalization and tensorialization properties. For the special case $K=0$ we have $\operatorname{CD}(0, N)=\operatorname{CD}^{*}(0, N)$. 
For arbitrary $K$ we have that any $\mathrm{CD}(K, N)$-space is also $\mathrm{CD}^{*}(K, N)$, but the converse implication is currently not perfectly understood (it is known that $\operatorname{CD}^{*}(K, N)$ implies $\operatorname{CD}\left(K^{*}, N\right)$, where $K^{*}=\frac{N-1}{N} K \leq K$ but the equivalence is open; for some recent progress see [7,8]).

From both the geometric and analytic perspective, a delicate issue concerning the $\mathrm{CD}(K, N)$ and $\mathrm{CD}^{*}(K, N)$ conditions is that they comprehend Finsler structures (see the last theorem in [34]), which after the works of Cheeger and Colding are known not to appear as Ricci-limit spaces.

To overcome this problem, in [2] the first author together with Ambrosio and Savaré introduced, specifically for the case $N=\infty$, a more restrictive condition which retains the stability properties with respect to measured Gromov-Hausdorff convergence and rules out Finsler geometries. This notion is called Riemannian curvature bound and denoted by $\operatorname{RCD}(K, \infty)$. According to the slightly finer axiomatization proposed by the authors and Ambrosio in [1], it can be presented as the reinforcement of the $\operatorname{CD}(K, \infty)$ condition with the requirement that the space is 'infinitesimally Hilbertian' (see also [19]), the latter meaning that the Sobolev space $W^{1,2}(X, \mathrm{~d}, \mathfrak{m})$ of real valued functions on $(X, \mathrm{~d}, \mathfrak{m})$ endowed with its canonical norm (i.e., the one built with the $L^{2}$ norm and the Cheeger energy) is Hilbert (in general it is only Banach).

In $[2,3]$ (see also [22] for the first progresses in this direction) it was shown that the $\operatorname{RCD}(K, \infty)$ condition is equivalent to the (properly written/understood) Bochner inequality

$$
\Delta \frac{|\nabla f|^{2}}{2} \geq \nabla f \cdot \nabla \Delta f+K|\nabla f|^{2} .
$$

The non-trivial refinement of this result to the finite dimensional case was carried out first by Erbar, Kuwada and Sturm [18] and slightly later with different techniques by the second author together with Ambrosio and Savaré in [4], where they proved that 'infinitesimal Hilbertianity plus $\mathrm{CD}^{*}(K, N)^{\prime}$ ' is equivalent to the Bochner inequality

$$
\Delta \frac{|\nabla f|^{2}}{2} \geq \frac{(\Delta f)^{2}}{N}+\nabla f \cdot \nabla \Delta f+K|\nabla f|^{2}
$$

(again, properly understood).

Although infinitesimal Hilbertianity is a requirement analytic in nature, the first author [21] showed that on infinitesimally Hilbertian $C^{*}(0, N)$-spaces the analog of the CheegerColding-Gromoll splitting theorem holds (see also [24] for the Abresch-Gromoll inequality), thus providing a geometric property which fails on general $\mathrm{CD}(K, N) / \mathrm{CD}^{*}(K, N)$-spaces. Unlike general $\mathrm{CD}(K, N) / \mathrm{CD}^{*}(K, N)$-spaces, infinitesimally Hilbertian $\mathrm{CD}^{*}(K, N)$-spaces are also known to be essentially non branching [31].

Still in the direction of understanding the geometric properties of infinitesimally Hilbertian $\mathrm{CD}^{*}(K, N)$-spaces, a natural conjecture is that on such setting the tangent spaces (i.e., pointed measured Gromov-Hausdorff limits of rescaled spaces) are Euclidean at almost every point. Moreover, like for the Ricci-limits, the tangents should be unique at almost every point. Here we make a step towards these conjectures by proving the following result:

Theorem 1.1. Let $K \in \mathbb{R}, 1 \leq N<\infty$ and $(X, \mathrm{~d}, \mathfrak{m})$ be an infinitesimally Hilbertian $\mathrm{CD}^{*}(K, N)$-space. Then at $\mathfrak{m}$-almost every $x \in X$ there exists $n \in \mathbb{N}, n \leq N$, such that

$$
\left(\mathbb{R}^{n}, \mathrm{~d}_{E}, \mathscr{L}_{n}, 0\right) \in \operatorname{Tan}(X, \mathrm{~d}, \mathfrak{m}, x),
$$

where $\mathrm{d}_{E}$ is the Euclidean distance and $\mathscr{L}_{n}$ is the $n$-dimensional Lebesgue measure normalized so that $\int_{B_{1}(0)} 1-|x| \mathrm{d} \mathscr{L}_{n}(x)=1$. 
Here $\operatorname{Tan}(X, \mathrm{~d}, \mathfrak{m}, x)$ denotes the collection of pointed measured Gromov-Hausdorff limits of rescaled spaces centered at $x$. Notice that the normalization of the limit measure expressed in the statement plays little role and depends only on the choice of renormalization of rescaled measures in the process of taking limits.

The idea for the proof of Theorem 1.1 is the one used by Cheeger and Colding in [10], namely to prove that $\mathfrak{m}$-a.e. point is the middle point of a non-constant geodesic, noticing that in the limit of blow-ups the space becomes an infinitesimally Hilbertian $C^{*}(0, N)$-space and the geodesics a line, then to use the splitting to factorize a direction. At this point it is a matter of proving that one can factorize enough dimensions to deduce that the limit is really Euclidean. In order to do so, Cheeger and Colding used some additional geometric information that is currently unavailable in the non-smooth setting: this is why we can't really prove that every tangent is Euclidean but only the existence of such tangent space.

Instead, we use a crucial idea of Preiss [29], adapted by Le Donne [25] to the metricmeasure setting, which states that on doubling metric-measure spaces 'tangents of tangents are tangents themselves', see Theorem 3.2 for the precise statement. Notice that we report the proof of such result because Le Donne stated the theorem for pointed Gromov-Hausdorff convergence, while we need it for the pointed measured Gromov-Hausdorff topology. Yet, such variant presents no additional difficulties so that we will basically just follow Le Donne's argument keeping track of the measures involved.

Finally, we remark that given that Theorem 1.1 is proved via such compactness argument, in fact we prove the following slightly stronger statement: for every sequence of scalings we have that for m-a.e. $x$ there exists a subsequence (possibly depending on $x$ ) converging to a Euclidean space.

Acknowledgement. The second author acknowledges the support of the ETH fellowship, part of the work was written when he was supported by the ERC grant GeMeTheNES directed by Prof. Luigi Ambrosio. The third author acknowledges the support of the Academy of Finland project no. 137528.

\section{Preliminaries}

2.1. Pointed metric measure spaces. The basic objects we will deal with throughout the paper are metric measure spaces and pointed metric measure spaces, m.m.s. and p.m.m.s. for short.

For our purposes, an m.m.s. is a triple $(X, \mathrm{~d}, \mathfrak{m})$ where $(X, \mathrm{~d})$ is a complete and separable metric space and $m$ is a boundedly finite (i.e., finite on bounded subsets) non-negative complete Borel measure on it.

We will mostly work under the assumption that the measure $\mathfrak{m}$ is boundedly doubling, i.e., such that

$$
0<\mathfrak{m}\left(B_{2 r}(x)\right) \leq C(R) \mathfrak{m}\left(B_{r}(x)\right), \quad \forall x \in X, r \leq R,
$$

for some positive constant $C(R)>0$ depending on the radius $R>0$. Notice that the map $C:(0, \infty) \rightarrow(0, \infty)$ can, and will, be taken non-decreasing. 
The bound (2.1) implies that supp $\mathfrak{m}=X$ and $\mathfrak{m} \neq 0$ and by iteration one gets

$$
\mathfrak{m}\left(B_{R}(a)\right) \leq \mathfrak{m}\left(B_{r}(x)\right)(C(R))^{\log _{2}\left(\frac{r}{R}\right)+2}, \quad \forall 0<r \leq R, a \in X, x \in B_{R}(a) .
$$

In particular, this shows that bounded subsets are totally bounded and hence that boundedly doubling spaces are proper.

A p.m.m.s. is a quadruple $(X, \mathrm{~d}, \mathfrak{m}, \bar{x})$ where $(X, \mathrm{~d}, \mathfrak{m})$ is a metric measure space and $\bar{x} \in \operatorname{supp}(\mathfrak{m})$ is a given 'reference' point. Two p.m.m.s. $(X, \mathrm{~d}, \mathfrak{m}, \bar{x}),\left(X^{\prime}, \mathrm{d}^{\prime}, \mathfrak{m}^{\prime}, \bar{x}^{\prime}\right)$ are declared isomorphic provided there exists an isometry $T:(\operatorname{supp}(\mathfrak{m}), d) \rightarrow\left(\operatorname{supp}\left(\mathfrak{m}^{\prime}\right), d^{\prime}\right)$ such that $T_{\sharp} \mathfrak{m}=\mathfrak{m}^{\prime}$ and $T(\bar{x})=\bar{x}^{\prime}$.

We say that a p.m.m.s. $(X, \mathrm{~d}, \mathfrak{m}, \bar{x})$ is normalized provided $\int_{B_{1}(\bar{x})} 1-\mathrm{d}(\cdot, \bar{x}) \mathrm{d} \mathfrak{m}=1$. Obviously, given any p.m.m.s. $(X, \mathrm{~d}, \mathfrak{m}, \bar{x})$ there exists a unique $c>0$ such that $(X, \mathrm{~d}, c \mathfrak{m}, \bar{x})$ is normalized, namely $c:=\left(\int_{B_{1}(\bar{x})} 1-\mathrm{d}(\cdot, \bar{x}) \mathrm{dm}\right)^{-1}$.

We shall denote by $\mathcal{M}_{C(\cdot)}$ the class of (isomorphism classes of) normalized p.m.m.s. fulfilling (2.1) for given non-decreasing $C:(0, \infty) \rightarrow(0, \infty)$.

2.2. Pointed measured Gromov-Hausdorff topology and measured tangents. The definition of convergence of p.m.m.s. that we shall adopt is the following (see $[6,23,34]$ ):

Definition 2.1 (Pointed measured Gromov-Hausdorff convergence). A sequence $\left(X_{j}, \mathrm{~d}_{j}, \mathfrak{m}_{j}, \bar{x}_{j}\right)$ is said to converge in the pointed measured Gromov-Hausdorff topology (p-mGH for short) to $\left(X_{\infty}, \mathrm{d}_{\infty}, \mathfrak{m}_{\infty}, \bar{x}_{\infty}\right)$ if and only if there exists a separable metric space $\left(Z, \mathrm{~d}_{Z}\right)$ and isometric embeddings $\left\{\iota_{j}:\left(\operatorname{supp}\left(\mathfrak{m}_{j}\right), \mathrm{d}_{j}\right) \rightarrow\left(Z, \mathrm{~d}_{Z}\right)\right\}_{i \in \overline{\mathbb{N}}}$ such that for every $\varepsilon>0$ and $R>0$ there exists $i_{0}$ such that for every $i>i_{0}$

$$
\iota_{\infty}\left(B_{R}^{X_{\infty}}\left(\bar{x}_{\infty}\right)\right) \subset B_{\varepsilon}^{Z}\left[\iota_{j}\left(B_{R}^{X_{j}}\left(\bar{x}_{j}\right)\right)\right] \text { and } \iota_{j}\left(B_{R}^{X_{j}}\left(\bar{x}_{j}\right)\right) \subset B_{\varepsilon}^{Z}\left[\iota_{\infty}\left(B_{R}^{X_{\infty}}\left(\bar{x}_{\infty}\right)\right)\right],
$$

where $B_{\varepsilon}^{Z}[A]:=\left\{z \in Z: \mathrm{d}_{Z}(z, A)<\varepsilon\right\}$ for every subset $A \subset Z$, and

$$
\int_{Y} \varphi \mathrm{d}\left(\left(\iota_{j}\right)_{\sharp}\left(\mathfrak{m}_{j}\right)\right) \rightarrow \int_{Y} \varphi \mathrm{d}\left(\left(\iota_{\infty}\right)_{\sharp}\left(\mathfrak{m}_{\infty}\right)\right) \quad \forall \varphi \in C_{b}(Z),
$$

where $C_{b}(Z)$ denotes the set of real valued bounded continuous functions with bounded support in $Z$.

Sometimes in the following, for simplicity of notation, we will identify the spaces $X_{j}$ with their isomorphic copies $\iota_{j}\left(X_{j}\right) \subset Z$.

It is obvious that this is in fact a notion of convergence for isomorphism classes of p.m.m.s., the following proposition also follows by standard means, see, e.g., [23] for details.

Proposition 2.2. Let $C:(0, \infty) \rightarrow(0, \infty)$ be a non-decreasing function. Then there exists a distance $\mathscr{D}_{C(\cdot)}$ on $\mathcal{M}_{C(\cdot)}$ for which converging sequences are precisely those converging in the p-mGH sense. Furthermore, the space $\left(\mathcal{M}_{C(\cdot)}, D_{C(\cdot)}\right)$ is compact.

Notice that the compactness of $\left(\mathcal{M}_{C(\cdot)}, \mathscr{D}_{C(\cdot)}\right)$ follows by the standard argument of Gromov: the measures of spaces in $\mathcal{M}_{C(\cdot)}$ are uniformly boundedly doubling, hence balls of given radius around the reference points are uniformly totally bounded and thus compact in 
the Gromov-Hausdorff topology. Then weak compactness of the measures follows using the doubling condition again and the fact that they are normalized.

The objects of study of this paper are measured tangents, which are defined as follows. Let $(X, \mathrm{~d}, \mathfrak{m})$ be an m.m.s., $\bar{x} \in \operatorname{supp}(\mathfrak{m})$ and $r \in(0,1)$; we consider the rescaled and normalized p.m.m.s. $\left(X, r^{-1} \mathrm{~d}, \mathfrak{m}_{r}^{\bar{x}}, \bar{x}\right)$ where the measure $\mathfrak{m}_{r}^{\bar{x}}$ is given by

$$
\mathfrak{m}_{r}^{\bar{x}}:=\left(\int_{B_{r}(\bar{x})} 1-\frac{1}{r} \mathrm{~d}(\cdot, \bar{x}) \mathrm{d} \mathfrak{m}\right)^{-1} \mathfrak{m} .
$$

Then we define:

Definition 2.3 (The collection of tangent spaces $\operatorname{Tan}(X, \mathrm{~d}, \mathfrak{m}, \bar{x}))$. Let $(X, \mathrm{~d}, \mathfrak{m})$ be an m.m.s. and $\bar{x} \in \operatorname{supp}(\mathfrak{m})$. A p.m.m.s. $\left(Y, \mathrm{~d}_{Y}, \mathfrak{m}, y\right)$ is called a tangent to $(X, \mathrm{~d}, \mathfrak{m})$ at $\bar{x} \in X$ if there exists a sequence of radii $r_{i} \downarrow 0$ so that $\left(X, r_{i}^{-1} \mathrm{~d}, \mathfrak{m}_{r_{i}}^{\bar{x}}, \bar{x}\right) \rightarrow\left(Y, \mathrm{~d}_{Y}, \mathfrak{n}, y\right)$ as $i \rightarrow \infty$ in the pointed measured Gromov-Hausdorff topology.

We denote the collection of all the tangents of $(X, \mathrm{~d}, \mathfrak{m})$ at $\bar{x} \in X$ by $\operatorname{Tan}(X, \mathrm{~d}, \mathfrak{m}, \bar{x})$.

Notice that if $(X, \mathrm{~d}, \mathfrak{m})$ satisfies $(2.1)$ for some non-decreasing $C:(0, \infty) \rightarrow(0, \infty)$, then $\left(X, r^{-1} \mathrm{~d}, \mathfrak{m}_{r}^{x}, \bar{x}\right) \in \mathcal{M}_{C(\cdot)}$ for every $\bar{x} \in X$ and $r \in(0,1)$ and hence the compactness stated in Proposition 2.2 ensures that the $\operatorname{set} \operatorname{Tan}(X, \mathrm{~d}, \mathfrak{m}, \bar{x})$ is non-empty.

It is also worth noting that the map

$$
\operatorname{supp}(\mathfrak{m}) \ni x \mapsto\left(X, \mathrm{~d}, \mathfrak{m}_{r}^{x}, x\right)
$$

is (sequentially) $\mathrm{d}$-continuous for every $r>0$, the target space being endowed with the p-mGH convergence.

2.3. Lower Ricci curvature bounds. Here we quickly recall those basic definitions and properties of spaces with lower Ricci curvature bounds that we will need later on.

We denote by $\mathcal{P}(X)$ the space of Borel probability measures on the complete and separable metric space $(X, \mathrm{~d})$ and by $\mathcal{P}_{2}(X) \subset \mathcal{P}(X)$ the subspace consisting of all the probability measures with finite second moment.

For $\mu_{0}, \mu_{1} \in \mathcal{P}_{2}(X)$ the quadratic transportation distance $W_{2}\left(\mu_{0}, \mu_{1}\right)$ is defined by

$$
W_{2}^{2}\left(\mu_{0}, \mu_{1}\right)=\inf _{\gamma} \int_{X} \mathrm{~d}^{2}(x, y) \mathrm{d} \gamma(x, y),
$$

where the infimum is taken over all $\gamma \in \mathcal{P}(X \times X)$ with $\mu_{0}$ and $\mu_{1}$ as the first and the second marginal.

Assuming the space $(X, \mathrm{~d})$ to be geodesic, also the space $\left(\mathcal{P}_{2}(X), W_{2}\right)$ is geodesic. We denote by $\operatorname{Geo}(X)$ the space of (constant speed minimizing) geodesics on $(X, \mathrm{~d})$ endowed with the sup distance, and by $\mathrm{e}_{t}: \operatorname{Geo}(X) \rightarrow X, t \in[0,1]$, the evaluation maps defined by $\mathrm{e}_{t}(\gamma):=\gamma_{t}$. It turns out that any geodesic $\left(\mu_{t}\right) \in \operatorname{Geo}\left(\mathcal{P}_{2}(X)\right)$ can be lifted to a measure $\pi \in \mathcal{P}(\operatorname{Geo}(X))$, so that $\left(\mathrm{e}_{t}\right)_{\#} \pi=\mu_{t}$ for all $t \in[0,1]$. Given $\mu_{0}, \mu_{1} \in \mathcal{P}_{2}(X)$, we denote by $\operatorname{OptGeo}\left(\mu_{0}, \mu_{1}\right)$ the space of all $\pi \in \mathcal{P}(\operatorname{Geo}(X))$ for which $\left(\mathrm{e}_{0}, \mathrm{e}_{1}\right)_{\#} \pi$ realizes the minimum in (2.4). If $(X, \mathrm{~d})$ is geodesic, then the set $\operatorname{OptGeo}\left(\mu_{0}, \mu_{1}\right)$ is non-empty for any $\mu_{0}, \mu_{1} \in \mathcal{P}_{2}(X)$.

We turn to the formulation of the $\mathrm{CD}^{*}(K, N)$ condition, coming from [5], to which we also refer for a detailed discussion of its relation with the $\operatorname{CD}(K, N)$ condition (see also [7,8]). 
Given $K \in \mathbb{R}$ and $N \in[1, \infty)$, we define the distortion coefficient

$$
[0,1] \times \mathbb{R}^{+} \ni(t, \theta) \mapsto \sigma_{K, N}^{(t)}(\theta)
$$

as

$$
\sigma_{K, N}^{(t)}(\theta):= \begin{cases}+\infty, & \text { if } K \theta^{2} \geq N \pi^{2}, \\ \frac{\sin (t \theta \sqrt{K / N})}{\sin (\theta \sqrt{K / N})} & \text { if } 0<K \theta^{2}<N \pi^{2}, \\ t & \text { if } K \theta^{2}=0, \\ \frac{\sinh (t \theta \sqrt{K / N})}{\sinh (\theta \sqrt{K / N})} & \text { if } K \theta^{2}<0 .\end{cases}
$$

Definition 2.4 (Curvature dimension bounds). Let $K \in \mathbb{R}$ and $N \in[1, \infty)$. We say that an m.m.s. $(X, \mathrm{~d}, \mathfrak{m})$ is a $\mathrm{CD}^{*}(K, N)$-space if for any two measures $\mu_{0}, \mu_{1} \in \mathcal{P}(X)$ with support bounded and contained in $\operatorname{supp}(\mathfrak{m})$ there exists a measure $\pi \in \operatorname{OptGeo}\left(\mu_{0}, \mu_{1}\right)$ such that for every $t \in[0,1]$ and $N^{\prime} \geq N$ we have

$$
\text { (2.5) }-\int \rho_{t}^{1-\frac{1}{N^{\prime}}} \mathrm{d} \mathfrak{m} \leq-\int \sigma_{K, N^{\prime}}^{(1-t)}\left(\mathrm{d}\left(\gamma_{0}, \gamma_{1}\right)\right) \rho_{0}^{-\frac{1}{N^{\prime}}}+\sigma_{K, N^{\prime}}^{(t)}\left(\mathrm{d}\left(\gamma_{0}, \gamma_{1}\right)\right) \rho_{1}^{-\frac{1}{N^{\prime}}} \mathrm{d} \boldsymbol{\pi}(\gamma),
$$

where for any $t \in[0,1]$ we have written $\left(\mathrm{e}_{t}\right)_{\sharp} \pi=\rho_{t} \mathfrak{m}+\mu_{t}^{S}$ with $\mu_{t}^{s} \perp \mathfrak{m}$.

Notice that if $(X, \mathrm{~d}, \mathfrak{m})$ is a $C D^{*}(K, N)$-space, then so is $(\operatorname{supp}(\mathfrak{m}), \mathrm{d}, \mathfrak{m})$, hence it is not restrictive to assume that $\operatorname{supp}(\mathfrak{m})=X$, a hypothesis that we shall always implicitly do from now on. It is also immediate to establish the following properties.

Property 1. If $(X, \mathrm{~d}, \mathfrak{m})$ is $\mathrm{CD}^{*}(K, N)$, then the same is true for $(X, \mathrm{~d}, c \mathrm{~m})$ for any $c>0$. If $(X, \mathrm{~d}, \mathfrak{m})$ is $\mathrm{CD}^{*}(K, N)$, then for $\lambda>0$ the space $(X, \lambda \mathrm{d}, \mathfrak{m})$ is $\operatorname{CD}^{*}\left(\lambda^{-2} K, N\right)$.

On $\mathrm{CD}^{*}(K, N)$ a natural version of the Bishop-Gromov volume growth estimate holds (see [5] for the precise statement), it follows that for any given $K \in \mathbb{R}, N \in[1, \infty)$ there exists a function $C:(0, \infty) \rightarrow(0, \infty)$ depending on $K, N$ such that any $\operatorname{CD}^{*}(K, N)$-space $(X, \mathrm{~d}, \mathfrak{m})$ fulfills $(2.1)$.

In order to enforce, in some weak sense, a Riemannian-like behavior of spaces with a curvature-dimension bound, a strengthening of the $\mathrm{CD}^{*}(K, N)$ has been proposed in [2] (see also $[1,4,18,19,28])$ : it consists in requiring that the space $(X, \mathrm{~d}, \mathfrak{m})$ is such that the Sobolev space $W^{1,2}(X, \mathrm{~d}, \mathfrak{m})$ is Hilbert, a condition we shall refer to as 'infinitesimal Hilbertianity'. It is out of the scope of this note to provide full details about the definition of $W^{1,2}(X, \mathrm{~d}, \mathfrak{m})$ and its relevance in connection with Ricci curvature lower bounds. We will instead be satisfied in recalling the definition and two crucial properties which are relevant for our discussion: the stability (see [23] and references therein) and the splitting theorem (see [21]).

First of all recall that on an m.m.s. there is not a canonical notion of 'differential of a function' $f$ but at least we can speak of 'modulus of the differential', called weak upper differential and denoted with $|D f|_{w}$; with this object one defines the Cheeger energy

$$
\mathrm{Ch}(f):=\frac{1}{2} \int_{X}|D f|_{w}^{2} \mathrm{dm} .
$$

The Sobolev space $W^{1,2}(X, \mathrm{~d}, \mathfrak{m})$ is by definition the space of $L^{2}(X, \mathfrak{m})$ functions having finite Cheeger energy, and it is endowed with the natural norm $\|f\|_{W^{1,2}}^{2}:=\|f\|_{L^{2}}^{2}+2 \mathrm{Ch}(f)$ 
which makes it a Banach space. We remark that, in general, $W^{1,2}(X, \mathrm{~d}, \mathfrak{m})$ is not Hilbert (for instance, on a smooth Finsler manifold the space $W^{1,2}$ is Hilbert if and only if the manifold is actually Riemannian); in case $W^{1,2}(X, \mathrm{~d}, \mathfrak{m})$ is Hilbert then we say that $(X, \mathrm{~d}, \mathfrak{m})$ is infinitesimally Hilbertian. Now we state two fundamental properties of infinitesimally Hilbertian $\mathrm{CD}^{*}(K, N)$ spaces.

Theorem 2.5 (Stability). Let $K \in \mathbb{R}$ and $N \in[1, \infty)$. Then the class of normalized p.m.m.s. $(X, \mathrm{~d}, \mathfrak{m}, \bar{x})$ such that $(X, \mathrm{~d}, \mathfrak{m})$ is infinitesimally Hilbertian and $\mathrm{CD}^{*}(K, N)$ is closed (hence compact) with respect to $p$ - $m G H$ convergence.

Theorem 2.6 (Splitting). Let $(X, \mathrm{~d}, \mathfrak{m})$ be an infinitesimally Hilbertian $\mathrm{CD}^{*}(0, N)$ space with $1 \leq N<\infty$. Suppose that $\operatorname{supp}(\mathfrak{m})$ contains a line. Then $(X, \mathrm{~d}, \mathfrak{m})$ is isomorphic to $\left(X^{\prime} \times \mathbb{R}, \mathrm{d}^{\prime} \times \mathrm{d}_{E}, \mathfrak{m}^{\prime} \times \mathscr{L}_{1}\right)$, where $\mathrm{d}_{E}$ is the Euclidean distance, $\mathscr{L}_{1}$ the Lebesgue measure and $\left(X^{\prime}, \mathrm{d}^{\prime}, \mathfrak{m}^{\prime}\right)$ is an infinitesimally Hilbertian $\mathrm{CD}^{*}(0, N-1)$-space if $N \geq 2$ and a singleton if $N<2$.

Notice that for the particular case $K=0$ the $\operatorname{CD}^{*}(0, N)$ condition is the same as the $\mathrm{CD}(0, N)$ one. Also, in the statement of the splitting theorem, by 'line' we intend an isometric embedding of $\mathbb{R}$.

Notice that Theorem 2.5 and Property 1 ensure that the following holds for any $K, N$.

Property 2. If $(X, \mathrm{~d}, \mathfrak{m})$ is an infinitesimally Hilbertian $\mathrm{CD}^{*}(K, N)$-space and $x \in X$, we have that every $(Y, \mathrm{~d}, \mathfrak{n}, y) \in \operatorname{Tan}(X, \mathrm{~d}, \mathfrak{m}, x)$ is infinitesimally Hilbertian and $\operatorname{CD}^{*}(0, N)$.

\section{Proof of the main result}

We will first show that at almost every point in a $\mathrm{CD}^{*}(K, N)$-space there exist a geodesic for which the point is an interior point. Then we prove that iterated tangents of $\mathrm{CD}^{*}(K, N)$ spaces are still tangents of the original space (actually we prove this part in the slightly more general framework of m.m.s. satisfying (2.1)). Finally, we use the interior points of geodesics and iterated tangents together with the splitting theorem (Theorem 2.6) to conclude the proof of Theorem 1.1.

3.1. Prolongability of geodesics. The following result is a simple consequence of the definition of $C D^{*}(K, N)$-space. The same argument was used in $[20,30]$, which were in turn inspired by some ideas in [26].

Lemma 3.1 (Prolongability of geodesics). Let $K \in \mathbb{R}, N \in[1, \infty)$ and $(X, \mathrm{~d}, \mathfrak{m})$ be a CD* $(K, N)$-space that is not a singleton. Then at $\mathfrak{m}$-almost every $x \in X$ there exists a non-constant geodesic $\gamma \in \operatorname{Geo}(X)$ so that $\gamma_{\frac{1}{2}}=x$.

Proof. Take $x_{0} \in X$ and $R>0$. Define

$$
\mu_{0}=\frac{1}{\mathfrak{m}\left(B_{R}\left(x_{0}\right)\right)} \mathfrak{m}\left\llcorner B_{R}\left(x_{0}\right) \quad \text { and } \quad \mu_{1}=\delta_{x_{0}} .\right.
$$


Let $\pi \in \operatorname{OptGeo}\left(\mu_{0}, \mu_{1}\right)$ be the measure satisfying (2.5). With the notation of (2.5) we then have

$$
-\int \rho_{t}^{1-\frac{1}{N}} \mathrm{dm} \leq-\int \sigma_{K, N}^{(1-t)}\left(\mathrm{d}\left(\gamma_{0}, \gamma_{1}\right)\right) \rho_{0}^{-\frac{1}{N}} \mathrm{~d} \pi(\gamma) \rightarrow-\mathfrak{m}\left(B_{R}\left(x_{0}\right)\right)^{\frac{1}{N}}, \quad \text { as } t \downarrow 0 .
$$

Let us write $E_{t}:=\left\{x \in X: \rho_{t}(x)>0\right\}$. By Jensen's inequality we get

$$
-\int \rho_{t}^{1-\frac{1}{N}} \mathrm{~d} \mathfrak{m}=-\int_{E_{t}} \rho_{t}^{1-\frac{1}{N}} \mathrm{~d} \mathfrak{m} \geq-\mathfrak{m}\left(E_{t}\right)\left(\frac{1}{\mathfrak{m}\left(E_{t}\right)} \int_{E_{t}} \rho_{t} \mathrm{~d} \mathfrak{m}\right)^{1-\frac{1}{N}} \geq-\mathfrak{m}\left(E_{t}\right)^{\frac{1}{N}} .
$$

Since the optimal transport is performed along geodesics connecting the points of $B_{R}\left(x_{0}\right)$ to $x_{0}$, we have the inclusion $E_{t} \subset B_{R}\left(x_{0}\right)$; therefore $\mathfrak{m}\left(E_{t}\right) \rightarrow \mathfrak{m}\left(B_{R}\left(x_{0}\right)\right)$ as $t \downarrow 0$, hence m-a.e. $x \in B_{R}\left(x_{0}\right)$ belongs to $E_{t}$ for some $t_{x}>0$. By construction, for m-a.e. $x \in E_{t}$ there exist $\gamma \in \operatorname{Geo}(X)$ and $t>0$ with $\gamma_{t}=x$ and $\gamma_{1}=x_{0}$, thus for m-a.e. $x \in B_{R}\left(x_{0}\right)$ there exists a non-constant geodesic $\gamma \in \operatorname{Geo}(X)$ so that $\gamma_{\frac{1}{2}}=x$. The conclusion follows by covering the space $X$ with countably many balls.

3.2. Tangents of tangents are tangents. In this subsection we adapt the celebrated theorem of Preiss [29] of iterated tangents of measures in $\mathbb{R}^{n}$ to our setting. In particular we are inspired by [25, Theorem 1.1], where Le Donne proved that for metric spaces with doubling measure almost everywhere the tangents of tangents are tangents of the original space. The difference here is that we also include the weak convergence of measures to the notion of tangents.

Theorem 3.2 ('Tangents of tangents are tangents'). Let $(X, \mathrm{~d}, \mathfrak{m})$ be an m.m.s. satisfying (2.1) for some $C:(0, \infty) \rightarrow(0, \infty)$. Then for $\mathfrak{m}$-a.e. $x \in X$ the following holds: for any $\left(Y, \mathrm{~d}_{Y}, \mathfrak{n}, y\right) \in \operatorname{Tan}(X, \mathrm{~d}, \mathfrak{m}, x)$ and any $y^{\prime} \in Y$ we have

$$
\operatorname{Tan}\left(Y, \mathrm{~d}_{Y}, \mathfrak{n}_{1}^{y^{\prime}}, y^{\prime}\right) \subset \operatorname{Tan}(X, \mathrm{~d}, \mathfrak{m}, x),
$$

the measure $\mathrm{n}_{1}^{y^{\prime}}$ being defined as in (2.3).

Proof. Before starting let us recall that in our convention $\operatorname{supp}(\mathfrak{m})=X$. We need to prove that

$$
\begin{aligned}
& \mathfrak{m}\left(\left\{x \in X: \text { there exist }\left(Y, \mathrm{~d}_{Y}, \mathfrak{m}, y\right) \in \operatorname{Tan}(X, \mathrm{~d}, \mathfrak{m}, x)\right.\right. \text { and } \\
& \left.\left.\qquad y^{\prime} \in Y \text { such that }\left(Y, \mathrm{~d}_{Y}, \mathfrak{n}_{1}^{y^{\prime}}, y^{\prime}\right) \notin \operatorname{Tan}(X, \mathrm{~d}, \mathfrak{m}, x)\right\}\right)=0 .
\end{aligned}
$$

This will follow if we can show that for all $k, m \in \mathbb{N}$ one has

$$
\begin{aligned}
\mathfrak{m}(\{x \in X: & \text { there exist }\left(Y, \mathrm{~d}_{Y}, \mathfrak{n}, y\right) \in \operatorname{Tan}(X, \mathrm{~d}, \mathfrak{m}, x) \text { and } y^{\prime} \in Y \text { such that } \\
& \mathrm{d}_{Y}\left(y, y^{\prime}\right) \leq m \text { and } \mathscr{D}_{C(\cdot)}\left(\left(Y, \mathrm{~d}_{Y}, \mathfrak{n}_{1}^{y^{\prime}}, y^{\prime}\right),\left(X, r^{-1} \mathrm{~d}, \mathfrak{m}_{r}^{x}, x\right)\right) \geq 2 k^{-1} \\
& \text { for all } \left.\left.r \in\left(0, m^{-1}\right)\right\}\right)=0 .
\end{aligned}
$$

Fix $k, m \in \mathbb{N}$ and notice that since $\left(\mathcal{M}_{C(\cdot)}, \mathscr{D}_{C(\cdot)}\right)$ is compact, it is also separable. Hence it is sufficient to show that for any closed set $U \subset \mathcal{M}_{C(\cdot)}$ with $\operatorname{diam}_{\mathscr{D}_{C(\cdot)}}(U)<(2 k)^{-1}$ the set

$$
\begin{aligned}
A=\{x \in X: & \text { there exist }\left(Y, \mathrm{~d}_{Y}, \mathfrak{n}, y\right) \in \operatorname{Tan}(X, \mathrm{~d}, \mathfrak{m}, x) \text { and } y^{\prime} \in Y \text { such that } \\
& \left(Y, \mathrm{~d}_{Y}, \mathfrak{n}_{1}^{y^{\prime}}, y^{\prime}\right) \in \mathcal{U}, \mathrm{d}_{Y}\left(y, y^{\prime}\right) \leq m \text { and } \\
& \left.\mathscr{D}_{C(\cdot)}\left(\left(Y, \mathrm{~d}_{Y}, \mathfrak{m}_{1}^{y^{\prime}}, y^{\prime}\right),\left(X, r^{-1} \mathrm{~d}, \mathfrak{m}_{r}^{x}, x\right)\right) \geq 2 k^{-1} \text { for all } r \in\left(0, m^{-1}\right)\right\}
\end{aligned}
$$


has $\mathfrak{m}$-measure zero. We start by proving that $A$ is Suslin, and thus $\mathfrak{m}$-measurable (this implies that the two subsets defined in the beginning of the proof are Suslin and thus m-measurable as well). To this aim, let $\mathcal{A} \subset X \times \mathcal{M}_{C(\cdot)}$ be given by the couples $\left(x,\left(Y, \mathrm{~d}_{Y}, \mathfrak{n}, y\right)\right)$ with $\left(Y, \mathrm{~d}_{Y}, \mathfrak{n}, y\right) \in \operatorname{Tan}(X, \mathrm{~d}, \mathfrak{m}, x)$ and recall that for every $r \in \mathbb{R}$ the map

$$
X \ni x \mapsto\left(X, r \mathrm{~d}, \mathfrak{m}_{1 / r}^{x}, x\right) \in\left(\mathcal{M}_{C(\cdot)}, \mathscr{D}_{C(\cdot)}\right)
$$

is continuous. Thus the set $\bigcup_{x \in X}\{x\} \times B_{1 / i}\left(X, r \mathrm{~d}, \mathfrak{m}_{1 / r}^{x}, x\right) \subset X \times \mathcal{M}_{C(\cdot)}$ is open and hence the identity

$$
\mathcal{A}=\bigcap_{i \in \mathbb{N}} \bigcap_{j \in \mathbb{N}} \bigcup_{r \geq j} \bigcup_{x \in X}\{x\} \times B_{1 / i}\left(X, r \mathrm{~d}, \mathfrak{m}_{1 / r}^{x}, x\right)
$$

shows that $\mathscr{A} \subset X \times \mathcal{M}_{C(\cdot)}$ is Borel. Next notice that the set $\mathcal{B} \subset \mathcal{M}_{C(\cdot)}$ defined by

$$
\begin{aligned}
\mathscr{B}:=\mathcal{U} \cap\left\{\left(Y, \mathrm{~d}_{Y}, \mathfrak{n}, \bar{y}\right):\right. & \mathscr{D}_{C(\cdot)}\left(\left(Y, \mathrm{~d}_{Y}, \mathfrak{n}, \bar{y}\right),\left(X, r^{-1} \mathrm{~d}, \mathfrak{m}_{r}^{x}, x\right)\right) \geq 2 k^{-1} \\
& \text { for all } \left.r \in\left(0, m^{-1}\right)\right\}
\end{aligned}
$$

is closed. Then using the fact that spaces in $\mathcal{M}_{C(\cdot)}$ are proper, it is easy to deduce that the set $e \subset \mathcal{M}_{C(\cdot)}$ given by

$$
\mathcal{C}:=\left\{\left(Y, \mathrm{~d}_{Y}, \mathfrak{n}, \bar{y}\right): \text { there exists } y^{\prime} \in Y \text { such that } \mathrm{d}_{Y}\left(y^{\prime}, \bar{y}\right) \leq m \text { and }\left(Y, \mathrm{~d}_{Y}, \mathfrak{n}_{1}^{y^{\prime}}, y^{\prime}\right) \in \mathscr{B}\right\}
$$

is closed as well. Hence, as $A$ is the projection on the first factor of $\mathcal{A} \cap(X \times \mathcal{C})$, it is Suslin, as claimed.

Now we proceed by contradiction and assume that for some $k, m, \mathcal{U}$ and $A$ as above one has $\mathfrak{m}(A)>0$. Let $a \in A$ be an $\mathfrak{m}$-density point of $A$, i.e.,

$$
\lim _{r \downarrow 0} \frac{\mathfrak{m}\left(A \cap B_{r}(a)\right)}{\mathfrak{m}\left(B_{r}(a)\right)}=1 .
$$

Since $a \in A$, there exist a space $\left(Y, \mathrm{~d}_{Y}, \mathfrak{n}, y\right) \in \operatorname{Tan}(X, \mathrm{~d}, \mathfrak{m}, a)$ and a point $y^{\prime} \in Y$ such that $\left(Y, \mathrm{~d}_{Y}, \mathfrak{n}_{1}^{y^{\prime}}, y^{\prime}\right) \in \mathcal{U}$. The fact that $\left(Y, \mathrm{~d}_{Y}, \mathfrak{n}, y\right) \in \operatorname{Tan}(X, \mathrm{~d}, \mathfrak{m}, a)$ grants the existence of a sequence $r_{i} \downarrow 0$ such that

$$
\left(X, r_{i}^{-1} \mathrm{~d}, \mathfrak{m}_{r_{i}}^{a}, a\right) \rightarrow\left(Y, \mathrm{~d}_{Y}, \mathfrak{n}, y\right) \quad \mathrm{p}-\mathrm{mGH} .
$$

Let $\left(Z, \mathrm{~d}_{Z}\right)$ be the separable metric space and $\iota_{i}, i \in \mathbb{N} \cup\{\infty\}$, the isometric embeddings given by the definition of $\mathrm{p}-\mathrm{mGH}$ convergence in (3.2). It is then immediate to check directly from Definition 2.1 that there exists a sequence $\left\{x_{i}\right\}_{i \in \mathbb{N}} \subset X$ such that

$$
\lim _{i \rightarrow \infty} \mathrm{d}_{Z}\left(\iota_{i}\left(x_{i}\right), \iota_{\infty}\left(y^{\prime}\right)\right)=0 .
$$

Notice that we have

$$
\begin{aligned}
& \varlimsup_{i \rightarrow \infty} \mathrm{d}_{Z}\left(\iota_{i}\left(x_{i}\right), \iota_{i}(a)\right) \\
& \quad \leq \varlimsup_{i \rightarrow \infty} \mathrm{d}_{Z}\left(\iota_{i}\left(x_{i}\right), \iota_{\infty}\left(y^{\prime}\right)\right)+\mathrm{d}_{Z}\left(\iota_{\infty}\left(y^{\prime}\right), \iota_{\infty}(y)\right)+\varlimsup_{i \rightarrow \infty} \mathrm{d}_{Z}\left(\iota_{\infty}(y), \iota_{i}(a)\right) \\
& \quad=\mathrm{d}_{Z}\left(\iota_{\infty}\left(y^{\prime}\right), \iota_{\infty}(y)\right),
\end{aligned}
$$

and thus by the definition of rescaled metrics we get

$$
\mathrm{d}\left(a, x_{i}\right) \leq C^{\prime} r_{i}, \quad \forall i \in \mathbb{N},
$$

for some constant $C^{\prime}>0$. 
Claim. There exists a sequence $\left\{a_{i}\right\}_{i \in \mathbb{N}} \subset A \subset X$ such that

$$
\lim _{i \rightarrow \infty} \mathrm{d}_{Z}\left(\iota_{i}\left(a_{i}\right), \iota_{\infty}\left(y^{\prime}\right)\right)=0 .
$$

Proof of the Claim. Here we use the fact that $\mathfrak{m}$ is locally doubling. This assumption is needed to deduce that for every $\varepsilon>0$ there exists $i_{0} \in \mathbb{N}$ such that for every $i \geq i_{0}$ we have $A \cap B_{\varepsilon r_{i}}\left(x_{i}\right) \neq \emptyset$. Indeed if it is not the case, there exists $\varepsilon_{0}>0$ such that

$$
A \cap B_{\varepsilon_{0} r_{i}}\left(x_{i}\right)=\emptyset, \quad \forall i \in J,
$$

for some infinite set of indexes $J \subset \mathbb{N}$. Up to choosing a smaller $\varepsilon_{0}$, (3.4) implies that $B_{\varepsilon_{0} r_{i}}\left(x_{i}\right) \subset B_{2 C^{\prime} r_{i}}(a)$ and so by estimate (2.2) we get

$$
\mathfrak{m}\left(B_{\varepsilon_{0} r_{i}}\left(x_{i}\right)\right) \geq C^{\prime \prime} \mathfrak{m}\left(B_{2 C r_{i}}(a)\right), \quad \forall i \in \mathbb{N},
$$

for some constant $C^{\prime \prime}$ independent of $i$ (but possibly depending on all other objects). Combining (3.6), (3.7) we get

$$
\mathfrak{m}\left(A \cap B_{2 C r_{i}}(a)\right) \leq \mathfrak{m}\left(B_{2 C r_{i}}(a) \backslash B_{\varepsilon_{0} r_{i}}\left(x_{i}\right)\right) \leq\left(1-C^{\prime \prime}\right) \mathfrak{m}\left(B_{2 C r_{i}}(a)\right), \quad \forall i \in J,
$$

and thus

$$
\lim _{i \rightarrow \infty} \frac{\mathfrak{m}\left(A \cap B_{2 C r_{i}}(a)\right)}{\mathfrak{m}\left(B_{2 C r_{i}}(a)\right)} \leq 1-C^{\prime \prime}<1
$$

contradicting that $a$ is an $\mathfrak{m}$-density point of $A$, namely (3.1).

Hence for every $\varepsilon>0$ eventually it holds $A \cap B_{\varepsilon r_{i}}\left(x_{i}\right) \neq \emptyset$. With a diagonalization argument we can then find a sequence $\left(a_{i}\right) \subset A$ such that $\lim _{i \rightarrow \infty} r_{i}^{-1} \mathrm{~d}\left(a_{i}, x_{i}\right)=0$. Recalling that $\mathrm{d}_{Z}\left(\iota_{i}\left(a_{i}\right), \iota_{i}\left(x_{i}\right)\right)=r_{i} \mathrm{~d}\left(a_{i}, x_{i}\right)$, the Claim follows from (3.3).

By (3.5) and directly from the definition of p-mGH convergence, using the same space $\left(Z, d_{Z}\right)$ and the same embeddings $\iota_{i}$ granting the convergence in (3.2) we deduce that

$$
\left(X, r_{i}^{-1} \mathrm{~d}, \mathrm{~m}_{r_{i}}^{a}, a_{i}\right) \rightarrow\left(Y, \mathrm{~d}_{Y}, \mathfrak{n}, y^{\prime}\right) \quad \mathrm{p}-\mathrm{mGH} .
$$

Since in the normalization (2.3) we use functions of the form $\chi_{B_{r}(\bar{x})}(\cdot)\left(1-\mathrm{d}_{Z}(\cdot, \bar{x})\right) \in C_{b}$, from weak convergence it follows that

$$
\left(X, r_{i}^{-1} \mathrm{~d}, \mathfrak{m}_{r_{i}}^{a_{i}}, a_{i}\right) \rightarrow\left(Y, \mathrm{~d}_{Y}, \mathfrak{n}_{1}^{y^{\prime}}, y^{\prime}\right) \quad \mathrm{p}-\mathrm{mGH},
$$

and thus for $i$ large enough

$$
\mathscr{D}_{C(\cdot)}\left(\left(X, r_{i}^{-1} \mathrm{~d}, \mathfrak{m}_{r_{i}}^{a_{i}}, a_{i}\right),\left(Y, \mathrm{~d}_{Y}, \mathfrak{n}_{1}^{y^{\prime}}, y^{\prime}\right)\right) \leq \frac{1}{2 k} .
$$

Since by construction $a_{i} \in A$, there exist spaces $\left(Y_{i}, \mathrm{~d}_{i}, \mathfrak{n}_{i}, y_{i}\right) \in \operatorname{Tan}\left(X, \mathrm{~d}, \mathfrak{m}, a_{i}\right)$ and points $y_{i}^{\prime} \in Y_{i}$ such that $\left(Y_{i}, \mathrm{~d}_{i},\left(\mathfrak{n}_{i}\right)_{1}^{y_{i}^{\prime}}, y_{i}^{\prime}\right) \in U$ and

$$
\mathscr{D}_{C(\cdot)}\left(\left(Y_{i}, \mathrm{~d}_{i},\left(\mathfrak{n}_{i}\right)_{1}^{y_{i}^{\prime}}, y_{i}^{\prime}\right),\left(X, r^{-1} \mathrm{~d}, \mathfrak{m}_{r}^{a_{i}}, a_{i}\right)\right) \geq \frac{2}{k} \quad \forall r \in\left(0, m^{-1}\right),
$$

where $\left(\mathfrak{n}_{i}\right)_{1}^{y_{i}^{\prime}}$ is the normalization of the measure $\mathfrak{n}_{i}$ at $y_{i}^{\prime}$ as in (2.3). 
Therefore by combining the bound $\operatorname{diam}_{\mathscr{D}_{C(\cdot)}}(\mathcal{U}) \leq(2 k)^{-1}$ with (3.8) and (3.9), for sufficiently large $i$ we have

$$
\begin{aligned}
& \frac{2}{k} \leq \mathscr{D}_{C(\cdot)}\left(\left(Y_{i}, \mathrm{~d}_{i},\left(\mathfrak{n}_{i}\right)_{1}^{y_{i}^{\prime}}, y_{i}^{\prime}\right),\left(X, r_{i}^{-1} \mathrm{~d}, \mathfrak{m}_{r_{i}}^{a_{i}}, a_{i}\right)\right) \\
& \leq \mathscr{D}_{C(\cdot)}\left(\left(Y_{i}, \mathrm{~d}_{i},\left(\mathfrak{n}_{i}\right)_{1}^{y_{i}^{\prime}}, y_{i}^{\prime}\right),\left(Y, \mathrm{~d}_{Y}, \mathfrak{n}_{1}^{y^{\prime}}, y^{\prime}\right)\right) \\
& +\mathscr{D}_{C(\cdot)}\left(\left(Y, \mathrm{~d}_{Y}, \mathfrak{n}_{1}^{y^{\prime}}, y^{\prime}\right),\left(X, r_{i}^{-1} \mathrm{~d}, \mathfrak{m}_{r_{i}}^{a_{i}}, a_{i}\right)\right) \\
& \leq \operatorname{diam}_{D_{C(\cdot)}}(U)+\mathscr{D}_{C(\cdot)}\left(\left(Y, \mathrm{~d}_{Y}, \mathfrak{n}_{1}^{y^{\prime}}, y^{\prime}\right),\left(X, r_{i}^{-1} \mathrm{~d}, \mathfrak{m}_{r_{i}}^{a_{i}}, a_{i}\right)\right) \\
& \leq \frac{1}{2 k}+\frac{1}{2 k}=\frac{1}{k}
\end{aligned}
$$

which is a contradiction.

\subsection{Iterating tangents to conclude.}

Proof of Theorem 1.1. Let $Z \subset X$ be the set of full m-measure where both Theorem 3.2 and Lemma 3.1 hold, and fix $x \in Z$. We will prove that there exists a tangent space to $x$ isomorphic to $\left(\mathbb{R}^{n}, \mathrm{~d}_{E}, \mathscr{L}_{n}, 0\right)$ for some $n \leq N$.

Thanks to Lemma 3.1 there exists a non-constant geodesic $\gamma \in \operatorname{Geo}(X)$ so that $\gamma_{\frac{1}{2}}=x$, therefore every tangent $\left(Y_{1}, \mathrm{~d}_{1}, \mathfrak{n}_{1}, y_{1}\right) \in \operatorname{Tan}(X, \mathrm{~d}, \mathfrak{m}, x)$ contains an isometric image of $\mathbb{R}$ going through the point $y_{1}$.

As a tangent of an infinitesimally Hilbertian $\mathrm{CD}^{*}(K, N)$-space, $\left(Y_{1}, \mathrm{~d}_{1}, \mathfrak{n}_{1}\right)$ is an infinitesimally Hilbertian $C D^{*}(0, N)$-space (see Property 2$)$ and so by Theorem 2.6 it splits into $\left(\mathbb{R} \times X_{1}, \mathrm{~d}_{E} \times \mathrm{d}_{1}^{\prime}, \mathscr{L}_{1} \times \mathfrak{m}_{1}\right)$ with $\left(X_{1}, \mathrm{~d}_{1}^{\prime}, \mathfrak{m}_{1}\right)$ infinitesimally Hilbertian $\mathrm{CD}^{*}(0, N-1)$-space.

If $X_{1}$ is not a singleton, then it contains a point $x_{1}$ where again both Theorem 3.2 and Lemma 3.1 can be used. Therefore, again by Theorem 2.6, every pointed tangent space $\left(Y_{2}, \mathrm{~d}_{2}, \mathfrak{n}_{2}, y_{2}\right) \in \operatorname{Tan}\left(X_{1}, \mathrm{~d}_{1}, \mathfrak{m}_{1}, x_{1}\right)$ splits as $\left(\mathbb{R} \times X_{2}, \mathrm{~d}_{E} \times \mathrm{d}_{2}^{\prime}, \mathscr{L}_{1} \times \mathfrak{m}_{2}\right)$ with $\left(X_{2}, \mathrm{~d}_{2}^{\prime}, \mathfrak{m}_{2}\right)$ infinitesimally Hilbertian $\mathrm{CD}^{*}(0, N-2)$-space.

By Theorem 2.6 this process can be iterated at most 'integer part of $N$ '-times before producing a space $X_{n}$. Tracing back the lines that have been factorized we conclude that $\left(\mathbb{R}^{n}, \mathrm{~d}_{E}, \mathscr{L}_{n}, 0\right) \in \operatorname{Tan}(X, \mathrm{~d}, \mathfrak{m}, x)$ for some $n \leq N$, as desired.

\section{References}

[1] L. Ambrosio, N. Gigli, A. Mondino and T. Rajala, Riemannian Ricci curvature lower bounds in metric spaces with $\sigma$-finite measure, Trans. Amer. Math. Soc., to appear.

[2] L. Ambrosio, N. Gigli and G. Savaré, Metric measure spaces with Riemannian Ricci curvature bounded from below, preprint 2011, http://arxiv.org/abs/1109.0222.

[3] L. Ambrosio, N. Gigli and G. Savaré, Bakry-Émery curvature-dimension condition and Riemannian Ricci curvature bounds, preprint 2012, http://arxiv .org/abs/1209.5786.

[4] L. Ambrosio, A. Mondino and G. Savaré, Nonlinear diffusion equations and curvature conditions in metric measure spaces, preprint 2013.

[5] K. Bacher and K.-T. Sturm, Localization and tensorization properties of the curvature-dimension condition for metric measure spaces, J. Funct. Anal. 259 (2010), 28-56.

[6] D. Burago, Y. Burago and S. Ivanov, A course in metric geometry, Grad. Stud. Math., American Mathematical Society, Providence 2001.

[7] F. Cavalletti, Decomposition of geodesics in the Wasserstein space and the globalization property, preprint 2012, http://arxiv.org/abs/1209.5909. 
[8] F. Cavalletti and K.-T. Sturm, Local curvature-dimension condition implies measure-contraction property, J. Funct. Anal. 262 (2012), 5110-5127.

[9] J. Cheeger and T. Colding, Lower bounds on Ricci curvature and the almost rigidity of warped products, Ann. of Math. 144 (1996), 189-237.

[10] J. Cheeger and T. Colding, On the structure of spaces with Ricci curvature bounded below I, J. Diff. Geom. 45 (1997), 406-480.

[11] J. Cheeger and T. Colding, On the structure of spaces with Ricci curvature bounded below II, J. Diff. Geom. 54 (2000), 13-35.

[12] J. Cheeger and T. Colding, On the structure of spaces with Ricci curvature bounded below III, J. Diff. Geom. 54 (2000), 37-74.

[13] T. Colding, Large manifolds with positive Ricci curvature, Invent. Math. 124 (1996), 193-214.

[14] T. Colding, Shape of manifolds with positive Ricci curvature, Invent. Math. 124 (1996), 175-191.

[15] T. Colding, Ricci curvature and volume convergence, Ann. of Math. 145 (1997), 477-501.

[16] T. Colding and A. Naber, Sharp Hölder continuity of tangent cones for spaces with a lower Ricci curvature bound and applications, Ann. of Math. 176 (2012), 1173-1229.

[17] T. Colding and A. Naber, Characterization of tangent cones of noncollapsed limits with lower Ricci bounds and applications, Geom. Funct. Anal. 23 (2013), 134-148.

[18] M. Erbar, K. Kuwada and K.-T. Sturm, On the equivalence of the entropic curvature-dimension condition and Bochner's inequality on metric measure spaces, preprint 2013, http://arxiv . org/abs/1303.4382.

[19] N. Gigli, On the differential structure of metric measure spaces and applications, preprint 2012, http:// arxiv.org/abs/1205.6622.

[20] N. Gigli, Optimal maps in non branching spaces with Ricci curvature bounded from below, Geom. Funct. Anal. 22 (2012), 990-999.

[21] N. Gigli, The splitting theorem in non-smooth context, preprint 2013, http://arxiv .org/abs/1302.5555.

[22] N. Gigli, K. Kuwada and S. Ohta, Heat flow on Alexandrov spaces, Comm. Pure Appl. Math. 66 (2013), 307-331.

[23] N. Gigli, A. Mondino and G. Savaré, A notion of pointed convergence of non-compact metric measure spaces and stability of Ricci curvature bounds and heat flows, preprint 2013.

[24] N. Gigli and S. Mosconi, The Abresch-Gromoll inequality in a non-smooth setting, Discrete Contin. Dyn. Syst., to appear.

[25] E. Le Donne, Metric spaces with unique tangents, Ann. Acad. Sci. Fenn. Math. 36 (2011), 683-694.

[26] J. Lott and C. Villani, Weak curvature conditions and functional inequalities, J. Funct. Anal. 245 (2007), 311-333.

[27] J. Lott and C. Villani, Ricci curvature for metric-measure spaces via optimal transport, Ann. of Math. 169 (2009), 903-991.

[28] A. Mondino, A new notion of angle between three points in a metric space, J. reine angew. Math., to appear.

[29] D. Preiss, Geometry of measures in $\mathbb{R}^{n}$ : Distribution, rectifiability, and densities, Ann. of Math. 125 (1987), 537-643.

[30] T. Rajala, Local Poincaré inequalities from stable curvature conditions on metric spaces, Calc. Var. Partial Differential Equations 44 (2012), 477-494.

[31] T. Rajala and K.-T. Sturm, Non-branching geodesics and optimal maps in strong $C D(K, \infty)$-spaces, Calc. Var. PDE, to appear.

[32] K.-T. Sturm, On the geometry of metric measure spaces. I, Acta Math. 196 (2006), 65-131.

[33] K.-T. Sturm, On the geometry of metric measure spaces. II, Acta Math. 196 (2006), 133-177.

[34] C. Villani, Optimal transport. Old and new, Grundlehren Math. Wiss. 338, Springer, Berlin 2009.

Nicola Gigli, Mathématiques, Université de Nice, Parc Valrose, 06108 Nice, France e-mail: nicola.gigli@unice.fr

Andrea Mondino, Departement Mathematik, ETH Zürich, Rämistrasse 101, 8092 Zürich, Switzerland

e-mail: andrea.mondino@math.ethz.ch

Tapio Rajala, Department of Mathematics and Statistics, P.O. Box, FI-40014 University of Jyväskylä, Finland e-mail: tapio.m.rajala@jyu.fi

Eingegangen 19. April 2013, in revidierter Fassung 9. Mai 2013 